Techniques \& Culture

\title{
Éclectisme et ethnologie
}

\section{Catherine Choron-Baix}

\section{OpenEdition \\ Journals}

Édition électronique

URL : https://journals.openedition.org/tc/2952

DOI : $10.4000 /$ tc. 2952

ISSN : 1952-420X

\section{Éditeur}

Éditions de l'EHESS

\section{Édition imprimée}

Date de publication : 12 décembre 2007

Pagination : 303-324

ISSN : 0248-6016

\section{Référence électronique}

Catherine Choron-Baix, «Éclectisme et ethnologie », Techniques \& Culture [En ligne], 48-49 | 2007, mis en ligne le 20 juin 2010, consulté le 29 septembre 2022. URL : http://journals.openedition.org/tc/2952 ; DOI : https://doi.org/10.4000/tc.2952 


\section{Catberine Choron-Baix*}

\section{Éclectisme et ethnologie}

La notion d'éclectisme a connu au cours de l'histoire des acceptions diverses, et fait l'objet de jugements de valeur controversés. Recentrée sur les mécanismes d'emprunts et d'assemblages qu'elle recouvre, elle paraît aujourd'hui appropriée pour caractériser les comportements et les formes d'expression modernes. Choisissant l'art contemporain comme observatoire privilégie de ce nouvel éclectisme ambiant, l'article interroge la manière dont l'ethnologie peut rendre compte de ces montages disparates qui relèvent à bien des égards des lois du syncrétisme, mais traduisent aussi les mobilités et une sorte d'ubiquité, intellectuelle et perceptive, que semblent favoriser les technologies de communication de masse et la généralisation des échanges.

Art contemporain, créativité, éclectisme, ethnologie, mondialisation, syncrétisme.

Ce sont quelques jalons d'une réflexion autour de l'éclectisme ou, plutôt, de "l'éclectique", considéré dans une vue extensive comme un donné anthropologique de nos sociétés modernes, qui sont ici esquissés ${ }^{1}$. La question est vaste et ne saurait être épuisée en quelques pages. Le propos est donc, modestement, didentifier quelques-uns des phénomènes d'assemblages hétéroclites qu'embrasse la notion, pour interroger la manière dont l'ethnologie peut en rendre compte. Propos exploratoire, plutôt épistémologique et méthodologique, qui se fonde sur des exemples, arbitrairement choisis, empruntés au monde de la création.

L'art paraît en effet le creuset de ces opérations de montages, où sadditionnent les techniques et les styles, et l'art contemporain tout

\footnotetext{
* CNRS, Laboratoire d'anthropologie urbaine, Ivry.

${ }^{1}$ Cet article fait suite à une journée d'étude sur le thème «Eclectisme et processus créateur ", organisée au CNRS, à Ivry sur Seine, le 10 mai 2005. Pour consulter le programme de cette journée, cf. la rubrique Archives du Laboratoire d'anthropologie urbaine : http://ivry.cnrs.fr/au/
} 
particulièrement, qui, à partir de la fin des années $1960^{2}$, décloisonne toujours plus les disciplines, opère des glissements de l'une à l'autre et mêle les genres. Associant gestuelle et mouvement, travail de la voix, théâtre et musique, réalisations plastiques et visuelles, faisant une place prééminente au mode performatif, les œuvres qui relèvent de ce «label " 3 ont souvent pour caractéristique de brouiller les frontières disciplinaires. Elles affichent en outre une forte transculturalité, en adoptant et adaptant des thématiques et des systèmes formels venus d'Afrique, d'Asie, et des sociétés non occidentales plus largement. Une logique du composite et du multiculturel, malaisée à décrire au moyen de l'ethnographie, semble simposer dans l'art contemporain, qui manifeste de nouvelles dispositions esthétiques tout aussi complexes à décrypter du point de vue de l'ethnologie.

Ces processus d'appropriation, d'absorption, de reformulation de traits culturels ne sont certes pas nouveaux. L'histoire témoigne au contraire de leur régularité sur tous les continents. La religion en est le théâtre privilégié, et un sujet de prédilection pour les historiens, sociologues et anthropologues attentifs à ces faits. Plus rares, quoiquills connaissent ces dernières années un essor considérable ${ }^{4}$, des travaux existent dans le domaine de la culture matérielle et des arts. Serge Gruzinski, par exemple, a pu montrer dans le détail les effets de la conquête espagnole sur la création artistique et artisanale des populations d'Amérique, avec la mise en circulation d'œuvres entre les cours européennes, le début des collections d'objets (les mirabilia curiosa) et ce quil nomme joliment le "relookage" des productions indigènes (2004): une première mondialisation, qui met en présence des partenaires inégaux en droits et en pouvoirs et révèle déjà la dimension

\footnotetext{
2 Comme le souligne Raymonde Moulin, l'art contemporain ne se définit pas selon un critère strictement chronologique «et ne se confond pas avec la production des artistes vivants. Les spécialistes, ajoute-t-elle, historiens contemporanéistes, critiques d'art et conservateurs - ne dissocient pas la périodisation de la caractérisation esthétique des œuvres. Ils s'entendent pour situer la naissance de l'art contemporain dans la décennie 1960-1969» (2003: 39).

3 Le terme est de Raymonde Moulin, qui précise que le label « contemporain » prit le relais de celui d' «avantgarde » à la fin des années 1960 , pour désigner «à la fois des créations associées à la tradition moderne de rupture et les créations postmodernes, nourries de références à une histoire déconstruite, qui ont ouvert la voie au pluralisme culturel », op.cit. page 40.

4L'ouvrage collectif rassemblé par Arjun Appadurai (1986) a marqué une étape importante dans l'intérêt porté à ces questions, ainsi que, plus récemment, celui qu'a dirigé Fred Myers (2001). Voir aussi le numéro 170/2004 de L'Homme.
} 
politique des situations de contact et de la circulation des savoirs et des artefacts.

Ces dynamiques interculturelles atteignent toutefois de nos jours une ampleur inégalée, conséquence du développement des moyens de communication, de duplication, de reproduction, et de la planétarisation des échanges. Elles indiquent une éthique et des canons stylistiques et esthétiques en pleine mutation, qu'il n'est pas simple de déchiffrer tant ils sont évolutifs et changeants.

Le paradigme du bricolage proposé par Claude Lévi-Strauss (I962) et abondamment repris ensuite ${ }^{5}$ fait florès dans les années I960 et I970 pour appréhender ces processus. Il est relayé, depuis, par les images du mélange, du métissage, de l'hybridation ou de la créolisation, du collage et jusqu'à celle du «bris-collage» suggérée par André Mary pour caractériser une postmodernité faite de la « fascination pour le morcellement des systèmes de sens et $[\ldots]$ les ordres fragmentaires » (2000 : I72-I73).

Ce concert de termes, tous plus ou moins métaphoriques, traduit un flottement terminologique symptomatique de la difficulté à qualifier les transferts interculturels qui se développent aujourd'hui à l'échelle mondiale. Il en est un, parmi eux, l'éclectisme justement, qui, dans le champ de la création artistique en particulier, revient régulièrement, quoique de manière incidente. Invoqué par les critiques et historiens d'art, par les anthropologues et les sociologues pour souligner l'hétérogénéité des œuvres et la multiplicité des références de leurs auteurs, le vocable relève davantage du commentaire que de la spécification d'un phénomène en soi. L'éclectisme est envisagé moins pour lui-même, comme effet de style à part entière, que comme le produit de la logique de cumul. Il apparaît comme un donné implicite, contenu dans l'hybride, dont il découle nécessairement, presque naturellement.

Le terme, pourtant, se rattache étymologiquement à lidée de choix, eklegô, et renvoie à un acte, calculé, de sélection ${ }^{6}$. Compris dans ce sens par

\footnotetext{
5 Dans les écrits de Michel de Certeau (1974) notamment, mais également dans des travaux plus récents, comme ceux de Benoit Berthou, par exemple, qui en fait le pivot de son analyse dans un texte publié dans le cadre du XVe congrès international d'esthétique de Tokyo, et intitulé « Les moyens du bord. Art contemporain et bricolage » (2001). Consultable sur le site http://gremes.free.fr/Interventions/benoit.html.

${ }^{6}$ L'eklektikos est celui qui est apte à choisir, et fait les justes sélections au sein d'ensembles hétérogènes pour ensuite agencer ou ré-agencer les fragments retenus. L'Encyclopédie Philo-notions remarque que l'adjectif et le nom eklektikos en grec sont tardifs, au sens de «apte à choisir », mais que eklektos figure chez Platon (Lois 938
} 
les philosophies de l'Antiquité, puis par Victor Cousin au XIX ${ }^{\mathrm{e}}$ siècle, il fut utilisé en histoire de l'art et de l'architecture pour désigner le retour périodique aux styles du passé. Mais cette acception s'est peu à peu érodée. Pour le sens commun qui en a banalisé l'usage et perverti la signification, l'éclectisme évoque largeur d'esprit, ouverture à la diversité et presque tolérance'. Il peut aussi, s'agissant d'art, se charger de connotations plus négatives. Il signale alors une accumulation désordonnée qui confine à la confusion, ou s'entend comme une tendance à la copie, au plagiat, signe d'un déficit de créativité.

Par-delà ce flou sémantique, il est désormais repris, pour qualifier leurs œuvres, par des artistes de plus en plus nombreux, qui en assument les différents attendus. Le recyclage de l'existant, plutôt que l'originalité, le mélange des styles, l'adhésion à la technologie moderne, la contestation des idées d'autonomie esthétique et de pureté artistique, l'accent mis sur la localisation temporelle et spatiale plutôt que sur l'universel et l'éternel, une production plus collective qu'individuelle - où le collectif est en tous cas implicite, à travers la charge mémorielle dont elle est porteuse- tels sont les arguments avancés par les créateurs contemporains pour justifier les montages qui composent leurs travaux. L'assemblage est revendiqué comme procédé créatif et le disparate s’énonce comme convention esthétique.

Face à cette valorisation de l'appropriation et de la transfiguration dans la création, peut-être y a-t-il quelque intérêt à revenir à la notion d'éclectisme dans son acception première, et à la préférer, dans la perspective d'une anthropologie de l'art contemporain au moins, aux métaphores du métissage, de l'hybridation, ou du collage. Recentrée sur le principe du choix qu'elle contient à l'origine, elle peut retrouver sa dimension dynamique et signifier que la refonte de modèles exogènes relève d'une stratégie d’invention et

b) au sens de «choisi », du verbe eklegô, qui signifie « choisir » et aussi « prélever ». Le Dictionnaire de la langue philosophique Foulquié note de son côté que l'Alexandrin Potamon passe pour l'inventeur de l'éclectisme, le mot et l'idée ayant été ensuite repris par Victor Cousin. Extraits du site http://www.eklecticlibrairie.com/DefinitionEclectique.htlm

${ }^{7}$ Tous les dictionnaires font état de cette acception extensive de l'éclectisme. Le Robert, par exemple, le définit comme «une disposition d'esprit» qui s'oppose au sectarisme, le dictionnaire Hachette comme « une largeur d'esprit permettant d'accueillir toute idée avec compréhension ", celui de l'Académie française comme «une disposition de l'esprit qui consiste à se garder de toute vue étroite et systématique ». Le Trésor de la langue française y voit le « refus de tout choix exclusif » et note qu'il peut prendre un sens péjoratif.

Pour une présentation exhaustive de ces différentes définitions, voir le site http://www.eklecticlibrairie.com/DefinitionEclectique.htlm. 
repose sur des opérations de sélection. L'éclectisme pourrait ainsi n'être plus tenu pour un état de fait, mais pour une part active du procès de création, fondée sur le discernement et le calcul. La notion couvre par ailleurs un registre étendu de variations, incluant non seulement celles qui jouent sur les frontières culturelles, mais également celles qui croisent les époques, les disciplines et les techniques. Plus englobante que le concept de syncrétisme qui s'applique à des situations d'acculturation le plus souvent duelles, entre culture-souche et culture-cible, ou entre des ensembles toujours identifiés, elle peut rendre plus sûrement cette logique additionnelle tous azimuts et sans cesse renouvelée qui est la marque de l'art contemporain. Elle peut du même coup apporter quelque lumière sur le geste créateur lui-même, comme sur l'engagement esthétique, social, politique, des artistes.

L'argumentation suppose un retour sur l'histoire de la notion et ce qu'elle recouvre à chaque période de son utilisation. Elle doit ensuite, envisageant l'éclectique comme une forme exacerbée du travail syncrétique, en cerner les spécificités, et proposer des moyens, conceptuels et méthodologiques, de l'appréhender. Seulement alors est-il possible de replacer la démarche éclectique dans le procès de création pour, enfin, tenter une interprétation de sa systématisation dans lart contemporain et de ses significations plus largement anthropologiques.

\section{De l'impossible fusion à l'œecumène contemporain}

Le syncrétisme et l'éclectisme conçu comme son corollaire ont longtemps fait l'objet des plus vives critiques. Les $\mathrm{XVI}^{\mathrm{e}}$ et $\mathrm{XVII}{ }^{\mathrm{e}}$ siècles, rappelle André Mary, dénoncent l'«impossible synthèse ", en stigmatisent l'anomie et les impasses. Plus tard, évangélisateurs et anthropologues ont en partage la même « horreur du mélange » (Mary 2000 : 12).

Dans l'histoire de la pensée occidentale, cependant, le phénomène éclectique, entendu cette fois comme courant intellectuel et artistique, se reproduit périodiquement, à la Renaissance, au XVIII ${ }^{\mathrm{e}}$ siècle, et singulièrement au $\mathrm{XIX}^{\mathrm{e}}$, lorsque Victor Cousin, portant un regard nouveau sur le passé, y voit une manière de libéralisation et une révolution contre l’académisme. «Puisque l'exclusif nous a mal réussi jusqu’ici, déclare-t-il dans son Cours d'histoire de la philosophie moderne, essayons de l'esprit de réconciliation". L' «éclectisme éclairé» dont il se réclame, «qui, jugeant 
toutes les doctrines, leur emprunte ce qu'elles ont de commun et de vrai, néglige ce qu'elles ont d'opposé et de faux ", représente pour lui une rupture épistémologique et un accès à la modernité (I847: I2). Dans le même temps, en peinture et en architecture, l'éclectisme se marque par une tendance archaïsante, par la volonté d'un retour à l'ancien pour créer des œuvres nouvelles. C'est vrai de l'art hellénistique, qui produit une série de styles plus "néo" que "rétro" (néo-archaïque, néo-classique, néo-attique), où les modèles antérieurs sont rélaborés pour s'adapter au contexte contemporain. En architecture prédominent la réappropriation simultanée de styles historiques, et la diversité, jusqu'à l'excès.

Mais d'autres rapprochements encore se font jour au cours de cette période, particulièrement mis en scène par les expositions universelles. La première d'entre elles, à Londres, en I85I, montre l'alliance de l'art et de l'industrie, l'union du beau et de l'utile. Les suivantes accueillent des spectacles exotiques qui captivent et inspirent les créateurs européens. À travers ses références au passé - occidental - l'art du XIX $\mathrm{X}^{\mathrm{e}}$ siècle rassure une bourgeoisie en quête de racines autant qu'une aristocratie nostalgique de l'ancien régime. Par ses emprunts aux civilisations orientales, il répond aux aspirations coloniales de la société industrielle. L'éclectisme est alors un art d'évasion, un voyage dans le temps et l'espace, une façon de fuir le progrès technique et les productions de la machine.

Il a toutefois ses détracteurs. Considéré comme n’engendrant que des œuvres sans identité, il est attaqué pour son défaut de composition, pour son désordre. "L'éclectisme est la plaie de l'art " ${ }^{8}$, écrit Jean-Baptiste Lassus, qui proclame "lindispensable nécessité de l'unité " et fustige "une fusion impossible entre des formes qui hurleraient de se rencontrer parce qu'elles appartiennent à des arts différents! " 9 Perce ici la tension entre innovation formelle et cohérence qui traverse toute l'histoire de la notion, jusqu'à ce radical changement de ton, ces dernières décennies, lorsque se disent, à travers elle, la complexité du monde et le souci de concilier les différences. L'éclectique devient une réponse au défi de l'altérité, dans un espace planétaire où sont exaltés le baroque, la pensée métisse et la rencontre øecuménique des cultures.

\footnotetext{
${ }^{8}$ Cette phrase fut la devise de Jean-Baptiste Lassus pour la présentation de son projet de restauration de Notre Dame de la Treille à Lille, en 1854. (Leniaud 1992). Je veux ici remercier Alice Thomine de me l'avoir signalée.

${ }^{9}$ Citation reproduite par Jean-Michel Leniaud, (1992: 244).
} 
L'art contemporain se situe dans cette veine, reproduisant, en le poussant à ses limites, le modèle syncrétique étudié par l'anthropologie de l'acculturation, sans toutefois développer toujours des formes pérennes.

\section{Des syncrétismes ponctuels}

En effet, si l'éclectisme moderne et postmoderne emporte l'adhésion des artistes et de leurs publics, ses retombées sociales restent généralement marginales. La fusion quill réalise vaut pour l'œuvre, le temps de l'œuvre, provisoire. Elle ne se stabilise et ne simpose dans le corps social que dans des circonstances particulières, historiquement déterminées. Le domaine musical fournit, il est vrai, un certain nombre d'exemples illustres du phénomène. Mais pour la masse des œuvres produites aujourd'hui, le mélange revêt un caractère ponctuel, aux effets limités, qui le différencie de celui qui opère dans les situations durables de contact qu'ont analysées Melville Herskovits et Roger Bastide au sein des sociétés créoles. Il procède néanmoins, à bien des égards, des lois du travail syncrétique mises en évidence par ces auteurs.

Le paradigme de la réinterprétation et celui de l'analogie que ces derniers identifièrent comme constitutifs du processus acculturatif ${ }^{10}$ semblent ainsi pouvoir s'appliquer pleinement à la démarche éclectique. L'artiste qui recycle réinvestit des contenus exogènes quil retient en raison de leur homologie avec ses propres catégories de pensée, en raison de leur contiguïté avec ses préoccupations. Une grande part des musiques populaires actuelles illustre ce mécanisme, et l'expérience de Jah Wobble en fournit un exemple parmi quantité d’autres. Issu du mouvement punk et ska des années I970-I980, ce bassiste anglais ne tarde pas à devenir une figure internationale de la scène dub $^{11}$, genre musical qui reprend les bases du reggae jamaïcain et les associe à d'autres sonorités, l'écho, la réverbération et des extraits de musiques extra-

\footnotetext{
10 Ces auteurs, comme le souligne André Mary, insistent sur le fait qu'il n'y a pas de syncrétisme sans réinterprétation, c'est-à-dire sans « appropriation des contenus culturels exogènes par le biais des catégories de pensée de la culture native » (Mary 2000 : 32). L'analogie, quant à elle, procède de ces « relations en miroir » qui posent entre les ensembles culturels des systèmes de correspondances ou d'équivalence fonctionnelle (ibid. 35).

${ }^{11}$ Le dub est né dans les studios de Kingston dans les années 1970 et s'est développé dans les dance halls de la Jamaïque. Il s'est exporté vers les États-Unis puis vers l'Angleterre, où il a subi l'influence du mouvement punk, et a ouvert la voie à la musique de rap, à la house music et aux courants électroniques.
} 
européennes. Réarrangeant des fragments narratifs pour créer des morceaux inédits, Jah Wobble s'adjoint la collaboration de musiciens d'horizons les plus divers, spécialistes de traditions celtes, chinoises, indiennes et, récemment, lao. En 2000 , il réalise un disque et donne de nombreux concerts, en Grande-Bretagne, en France, au Japon, avec une formation de musiciens et chanteurs originaires du Laos en exil en France ${ }^{12}$. Convaincu que le lam, leur art musical et vocal est, selon ses propres termes, «la musique rap de l'Asie du Sud-Est "13, il l'adapte à ses propres canons pour produire ce Molam Dub, un «mix " qui conjugue les voix asiatiques et la sonorité du khène, l'orgue-à-bouche lao, aux basses, boîtes à rythme et effets spéciaux des musiques "électroamplifiées " ${ }^{14}$. Il y a bien là "relations en miroir" entre des ensembles culturels qui sont dans des rapports d'équivalence ou sont, du moins, présentés comme tels.

Un autre des paramètres de l'anthropologie de l'acculturation, que Roger Bastide appelle le "principe de coupure", se vérifie dans lapproche éclectique. Il renvoie à l'alternance ou la coexistence, chez un même individu ou au sein d'un même groupe, de modèles en eux-mêmes irréductibles ${ }^{15}$. C'est lui qui permet à l'artiste ce va et vient entre des mondes distincts quil rapproche et rend tout à coup conciliables. Cette faculté de jouer sur les appartenances et les références, d'aller de l’une à l'autre, est ce qui, pour Roger Bastide, différencie l'hybride, synonyme à ses yeux de dégénérescence, de ce quill appelle «le syncrétisme en mosaïque » ${ }^{16}$, une mise en synergie qui ne va jamais jusqu'à la fusion et autorise une "dualité sans marginalité " (Bastide 1954: 499). Dans la démarche éclectique, le principe de coupure agit au maximum. Il y prend la forme d'une concomitance de références diverses, qui laisse à l'artiste une grande liberté de manœuvre, sans abandon de ses propres repères. Sans doute faut-il y voir une clé de l'approche

\footnotetext{
12 La formation Mo Lam Lao s'est constituée en France dans les années 1980, composée d'artistes originaires du Sud Laos réfugiés en France à la suite de la chute de la monarchie et de l'instauration de la République Démocratique Populaire du Laos. Les mo lam (de mo, maîtres, lam, genre poétique chanté du Laos méridional) sont auteurs interprètes de chants poétiques improvisés, et accompagnés au khène, un orgue-à-bouche en tuvaux de bambou. Pour une étude de cet art, voir Choron-Baix 1983 et 1992

13 Citation extraite du site Internet http://www.molamdub.com/dubmix.htm, créé à l'occasion de la sortie du disque Mo Lam Dub, en 2000.

${ }^{14}$ L'expression est de Marc Touché (2007).

${ }^{15}$ Pour un commentaire de cette notion, cf. André Mary $(2000: 37)$.

16 Pour un développement de cette notion, cf. Stefania Capone (2007).
} 
éclectique contemporaine, celle qui rend possibles les permutations entre différents ordres de représentation.

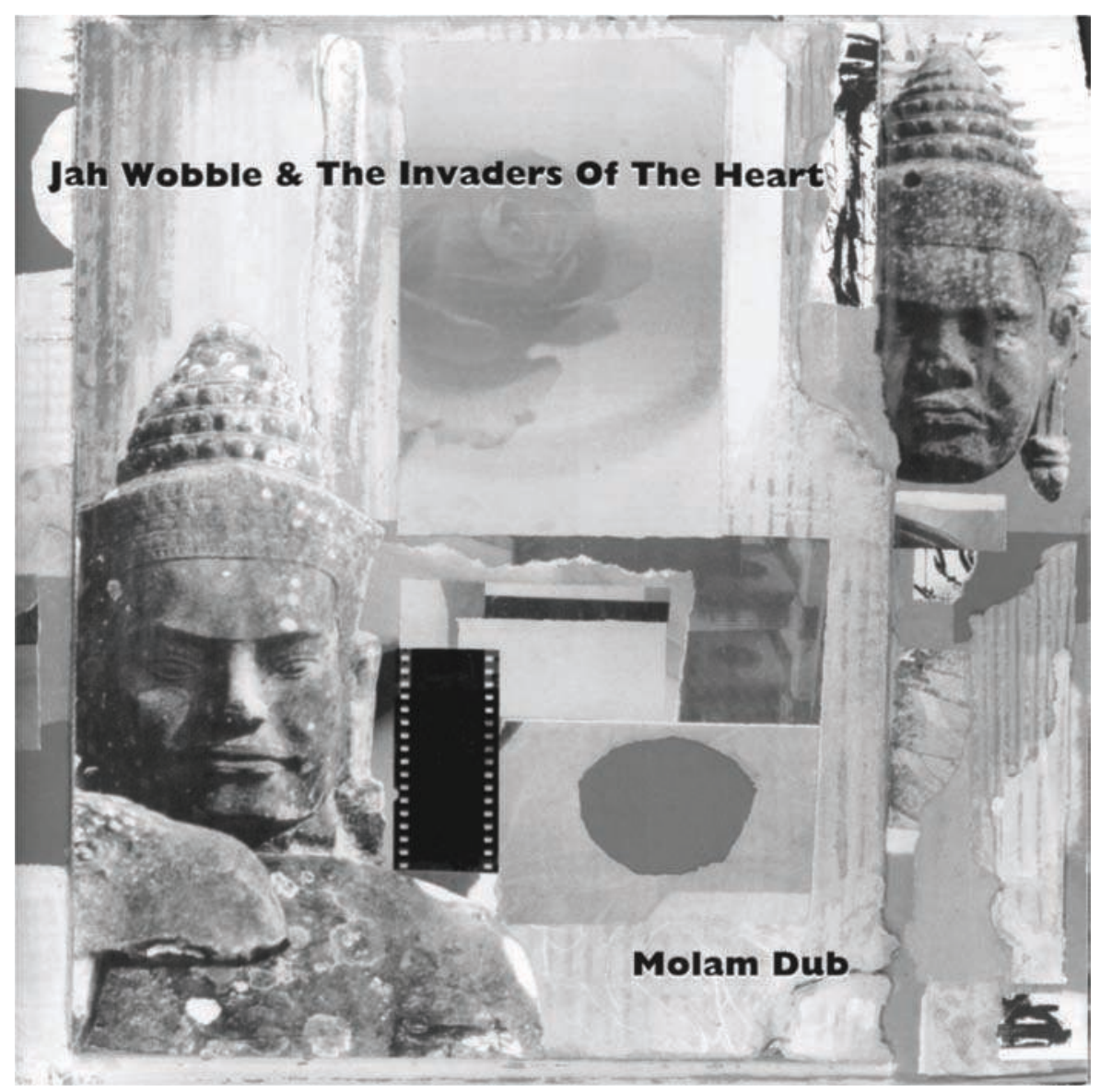

Figure 1. Pochette de l'album Molam Dub, de Jah Wobble \& The Invaders Of The Heart (C) 30 Hertz Records).

Reste la notion, majeure dans le paradigme du syncrétisme, de la "précontrainte", selon laquelle la matière symbolique récupérée demeure marquée par son usage antérieur. Elle a montré toute sa pertinence dans l’analyse des faits religieux, reliée à la problématique de la mémoire collective 
qui, fût-elle lacunaire, influe de manière décisive sur les conditions de transfert de fragments d'une tradition vers une autre. Sans un minimum de référence au passé, remarque André Mary, le bricolage n'est qu’une juxtaposition de bribes vides de sens (2000: 4I). Qu'advient-il, dans le champ de lart, de ces traces mémorielles et de ces matériaux pré-contraints, lorsqu'ils passent d'un contexte vers un autre ? La question est au cour de la compréhension du processus créateur, puisque la reprise consiste précisément, le plus souvent, à jouer de cette matière symbolique pour en déplacer le sens.

Soit l'exemple d'un artiste vietnamien-américain en résidence au Laos qui, impressionné par le choc de la modernisation dans ce pays, choisit quelques-uns de ses emblèmes les plus marqués, essentiellement tirés du registre religieux, pour traiter de cette thématique dans ses œuvres. Les hang lin, gouttières d'ondoiement des statues de bouddha, ou les bannières de prières plantées sur le champ de crémation à l'issue des cérémonies funéraires, éléments rituels au centre du bouddhisme lao, sont réinterprétées, dans ses propositions, comme des symboles de la confrontation entre tradition et modernité17. Le procédé est ici patent: isolant l'objet et l'extrayant de son cadre originel, l'artiste le détourne de ses usages pour servir une vision personnelle. C’est dans ce réemploi, inédit, que réside sa créativité.

Cette façon de faire n'appartient pas en propre aux créateurs contemporains. Pratiquée de tous temps, elle semble au contraire au fondement du geste artistique et ne se distingue aujourd'hui que par sa systématicité et sa plus grande visibilité.

\section{Un ressort de la créativité}

Les témoignages de cette inclination des artistes à puiser hors de leur temps et de leur environnement social et culturel les sources de leur inspiration abondent, et leurs collections privées en sont la plus évidente démonstration. Rodin, pour ne prendre qu'un exemple, accumula un ensemble hétéroclite d'œuvres de toutes provenances et de toutes époques, de l'Extrême et du Moyen-Orient, du Moyen Âge ou de l'époque classique, quil appelait ses «fragments de dieux». Il en recensait les matériaux et les techniques, en

17 Pour une présentation de ce travail, cf. le site de The Quiet in The Land. Art, Spirituality, Everyday Life, Luang Prabang 2004-2007, la mention concernant l'artiste Dinh Q. Lê, www.thequietintheland.org. 
étudiait les formes, pour les intégrer dans ses propres travaux. Dans les années I890-I900, rapporte Bénédicte Garnier, il se constitua un corpus monumental qui devint une des pièces maittresses de sa recherche (2002). Il juxtaposait des photographies d'antiques romains aux images de ses propres sculptures, ou découpait puis collait dans des carnets des dessins faits d'après les œuvres du passé, pour former de nouvelles compositions. Il reprit le jeu des confrontations, développé par Eugène Viollet-le-Duc au musée des arts comparés du Trocadéro quil fréquentait assidûment, et pratiquait l'association des similaires et des contraires. Il alla même plus loin dans la voie de l'éclectisme. "La vénération du sculpteur pour les maitres grees et romains, poursuit Bénédicte Garnier, ne l'empêcha pas d'exercer son esprit critique sur les antiques de sa collection. Il y gommait tout ce qui pouvait perturber son travail ou modifier le fil de sa pensée, et ne se privait pas de les remanier par le regard ou par l'ajout d'accessoires " (Ibid. : 57). Vers I895, il utilisait des objets préexistants quil transformait en matériaux de création, devançant les recherches de Picasso ou Henry Moore et de toutes ces générations d'artistes qui insèrent des objets du quotidien dans leurs œuvres. Il répétait de la sorte des exercices de composition dans lesquels entraient deux classes de matériaux, ses pièces de collection et ses propres figures moulées dans le plâtre ou en terre cuite. Son $\mathrm{Nu}$ féminin sur un alabastron égyptien, où l'antique sert de support à sa sculpture en équilibre instable, ou son Torse d'Eros en terre cuite fixé sur un vase en albâtre égyptien retourné, en sont de superbes illustrations ${ }^{18}$. Dans l'un et l'autre cas, l'objet hybride est assimilé par l'œil à une ouvre de Rodin. Ce dernier recourait ainsi aux procédés caractéristiques de l'éclectisme: juxtaposition, assemblage, rélaborations, manipulations, toutes motivées par les correspondances que lartiste décelait entre les créations auxquelles il empruntait et sa propre vision. Ainsi confia-t-il à propos des danseuses cambodgiennes quil découvrit à l'exposition coloniale de Marseille en 1906: «ce qui surtout m'étonnait et me ravissait, c'était de retrouver dans cet art d'Extrême-Orient, inconnu de moi jusqualors, les principes mêmes de l'art antique. (...) Tout ce que jadmirais dans les marbres antiques, ces Cambodgiennes me le donnaient, en y ajoutant l'inconnu et la souplesse de l'Extrême-Orient ${ }^{19}$. Dans ce dialogue avec l'antique et l'exotique, le sculpteur incarne la figure de

18 Voir les reproductions de ces œuvres dans Bénédicte Garnier $(2002: 62,63)$.

${ }^{19}$ Cité dans Rodin et l'Extrême-Orient (Collectif 1979 : 68). 
l'éclectique au XIX ${ }^{\mathrm{e}}$ siècle, qui rejette l'académisme et cherche hors de son environnement, en d'autres temps et d'autres lieux, la stimulation nécessaire à son inventivité.

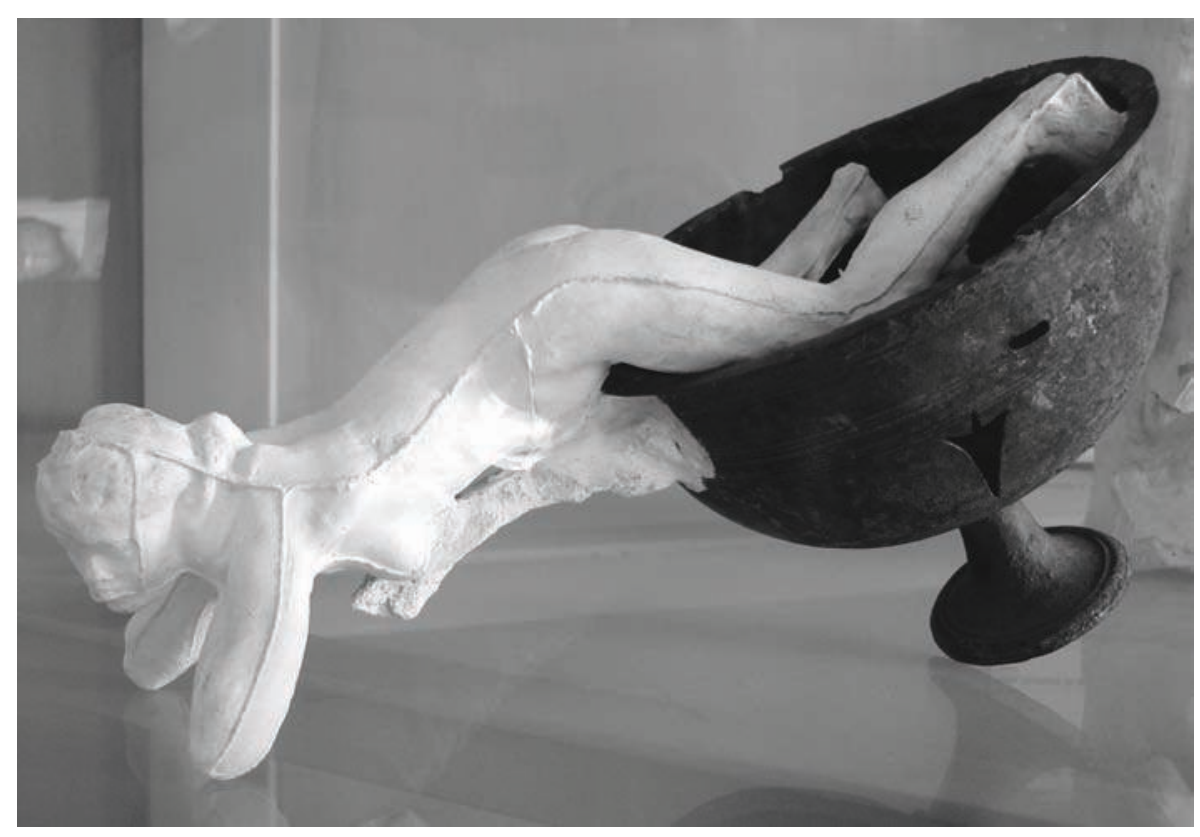

Figure 2. Auguste Rodin, «Assemblage : Petite faunesse dans une coupe en métal », plâtre et vase de bronze, 17,6 × 15 x 26 cm (S.372, (C) Musée Rodin, cliché de l'auteur).

Si elle annonce celle des modernes et des postmodernes, sa méthode de travail atteste surtout que le détour par l'ancien et l'autre lointain est un ressort essentiel de la créativité, ce que soulignent d'ailleurs, à différentes époques, différents auteurs. À la fin du XIX $\mathrm{X}^{\mathrm{e}}$ siècle, Gabriel Tarde, par exemple, note que c'est en développant des aptitudes au mimétisme que l'homme se montre innovant (Tarde 200I). Freud, après lui, dénonce la part d'illusion dans lidée de création. La prétendue imagination créatrice ne crée rien, affirme-t-il, elle ne fait que rassembler ce qui à l'origine était séparé 
(Freud 1987). Quant à Michel de Certeau, il fait du bricolage et du collage, de la combinaison et de la multiplication des compositions, l'essence du procès d’invention, précisant: «la créativité est l'art de réemployer et d’associer des matériaux hétérogènes " (1993:98).

Opération de sélection et de réagencement d'éléments épars, l'éclectisme est donc avant tout une démarche, un princeps méthodologique de lacte créateur, que notre époque cultive à l'extrême en puisant à une diversité toujours croissante de sources.

Cette réalité fut longtemps ignorée ou niée par l’idéologie de l'originalité. L'œuvre d'art se devait autrefois d'être unique, produit singulier d'une individualité hors du commun. Cette conception n'a plus cours aujourd'hui, et peu de créateurs contemporains se prévalent d’une propriété artistique exclusive, sortie, ex nihilo, de leur seul génie inventif. La plupart au contraire se déclarent en recherche, inscrits dans une filiation intellectuelle et artistique dont ils se reconnaissent comme des continuateurs. Ils ne prétendent pas produire du neuf mais travaillent plutôt à partir d'un stock mémoriel quils font évoluer. L'expérience du musicien de hip-hop est à cet égard très parlante. Dans le «mix " quill produit, il met sa touche personnelle à une matière sonore qui ne lui appartient pas mais dont il s'empare pour la transformer ${ }^{20}$. Il ne revendique pas une création mais plutôt un talent à remanier des éléments glanés auxquels il donne une destination nouvelle. Loriginalité, comme l'unité stylistique, ne sont plus des valeurs absolues (Shusterman 1992). L'art, désormais, s'assume comme appropriation et reformulation, et se pense en termes de collisions et de télescopages. Sous cette forme fractale, kaléidoscopique, il met à mal un certain nombre de postulats des sciences sociales et résiste à l'analyse.

\section{L'introuvable norme}

L'éclectisme contemporain se situe, tout d'abord, dans une temporalité complexe, à la fois retour sur le passé, valorisation de linstant et plongée dans le futur. Les emprunts dont il fait sa matière sont le plus souvent opportunistes, conjoncturels, fruits de contacts datés qui auraient pu ne pas

\footnotetext{
${ }^{20}$ Pour une description détaillée des opérations réalisées par le musicien dans le hip-hop, cf. Benoît Berthou, "Les moyens du bord. Art contemporain et bricolage", texte présenté au XVe congrès international d'esthétique, à Tokyo en août 2001, disponible sur Internet : http://gremes.free.fr/interventions/benoit.htlm.
} 
avoir lieu et qui peuvent demeurer sans suite. L'œuvre éclectique contient en elle une somme de possibles et l'évocation toujours un peu sous-jacente de ce qui n'est pas survenu, ou aurait pu arriver, ou peut encore arriver. Située dans une sorte de «présent perpétuel", elle tient de l'évènementiel, du surgissement sans précédent et sans lendemain, et se prête mal au récit historique (Bensa 2002). "L'œuvre d'un éclectique ne laisse pas de souvenir", disait Baudelaire (I846: I69). Dans linstallation, sa version contemporaine paroxystique et par définition temporaire, elle s'oppose, il est vrai, à la vision romantique de l'art stable et durable. Ici, l'œuvre se présente comme un agencement fugace procurant une émotion fugitive.

Le procédé éclectique se donne par ailleurs comme écart permanent. Imprévisible, il déjoue les typologies. Il offre peu de constantes et ne se soumet guère à la sériation, engendrant des œuvres qui ne peuvent être classifiées, si ce n'est comme ensembles fondamentalement composites, formés d'éléments hétérogènes qui, juxtaposés ou assemblés, perdent les propriétés et les significations qu’ils avaient chacun isolément et en revêtent de nouvelles.

L'éclectisme, dès lors, met en cause lidée même de style. Non pas que celui-ci soit un donné intemporel, immuable. Il se construit au contraire de manière continue, au long d’une chaîne de transmission qui fait appel à une mémoire des perceptions et des représentations esthétiques, et qui évolue suivant "un jeu de subtiles variations" (Martinelli 2005: 6). Mais dans la démarche éclectique, le principe de l'emprunt et du recyclage renouvelle sans cesse le système signalétique et ne renvoie plus à des " connotations partagées " (Martinet I968). Il devient difficile d'y détecter un langage, d’y reconnaitre des qualités distinctives. L'art contemporain nie la permanence de la structure, dont il pose au contraire la permutabilité des composantes. Et sil existe entre les œuvres un air de famille, celui-ci tient à leur parti pris et leur morphologie combinatoires plus qu’à des propriétés formelles communes. Le style éclectique est éminemment variable et c'est cette variabilité même, déclinable à l’infini, qui le définit.

C'est elle aussi qui en rend l'analyse délicate. Car pour le rendre intelligible dans ses fonctions sociales et symboliques, il faut en réduire l'effet de surprise, et rechercher, derrière l'apparente inconstance des formes, des lois de composition. Pour y parvenir, lethnographie doit se conduire dans plusieurs directions à la fois, du côté des acteurs -concepteurs et 
spectateurs- pour en retracer les parcours et les discours, en inventorier les répertoires de signes, et du côté des œuvres, dont elle doit faire l'autopsie et observer les modes de réception.

Dans cette investigation à visée totalisante, la biographie d'artiste fournit sans doute les matériaux les plus heuristiques. Alliant au récit d’une vie celui d'une trajectoire intellectuelle et réflexive, elle permet de contextualiser la création. Elle met à jour les rencontres, essentielles chez l'éclectique, qui font date dans son existence, et révèle les lieux qui font son univers. L'environnement urbain, contrasté, foisonnant, souvent violent, mais aussi le déplacement et le voyage, sont des motifs récurrents de l'art contemporain, qui renvoient au vécu des créateurs, et à ce quils tiennent pour des expériences fondatrices. Le portrait d'artiste met en lumière ces cheminements et ces temps forts et conduit au sens de l'ouvre, avec aussi ses "à côtés", inventaires d'objets et de souvenirs thésaurisés, d'archives personnelles qui dévoilent ses intérêts, ses interrogations, ses obsessions. L'approche biographique peut utilement se compléter du répertoire de ces matériaux de toutes sortes quil compile et ré-emploie dans ses travaux. On a vu limportance du «musée imaginaire » de Rodin dans sa recherche. Pour le sculpteur, "collectionner devint une seconde nature », un genre artistique en soi, indissociable du reste de sa création (Vilain 2002). Composée par et pour lui-même, sa collection est une introduction à son œuvre et à la découverte de sa personnalité. De même la "playlist » du musicien de hip-hop, cette sorte de banque de sons quil se constitue à partir de "bandes démos " 21 et de fichiers téléchargés sur Internet, est-elle un révélateur de ses choix de mixage. La collecte comme miroir de l'homme et de l'œuvre: la recension des données et objets que capitalise l'artiste éclaire ses intentions et sa méthode. Pas davantage que la biographie, pourtant, elle ne suffit à expliquer pourquoi les arts d'aujourd'hui sont si polyvalents et multiformes, pourquoi, dans leur élaboration puis dans leur réception, simpose cette esthétique du disparate.

\section{Art et politique}

Différentes interprétations de ce recours au passé, à l'exotisme et au bricolage dans la création contemporaine ont été avancées. La nostalgie d’un

${ }^{21}$ Morceaux de musique en attente de production. 
monde révolu, une vision enchantée de l'autre et la soif de ressourcement en sont des thèmes récurrents. Jean-Loup Amselle, commentant l'engouement actuel, sur le marché de l'art international, pour l'Afrique et ses artefacts, le tient pour un moyen de régénération d'un monde occidental en perte de repères (2005). André Mary, quant à lui, conçoit le collage postmoderne comme un syncrétisme "élémentaire ", simple assemblage de morceaux qui ne tient plus compte de la valeur mémorielle des ensembles où il puise. Il y voit le "symptôme de la mort de la structure ou d'un désespoir du sens " (2000: 4I). Ces analyses font un constat négatif: c'est une forme de désespérance, un épuisement du sens, qui motivent la démarche éclectique aujourd'hui.

D'autres raisons peuvent tout autant y pousser, plus réactives, et annonciatrices d'un changement d'habitus. Le refus de l'enfermement dans un style, dans une école, est un argument récurrent chez certains artistes. Le plasticien Bruno Peinado, auteur de l'exposition Perpetuum mobile, au Palais de Tokyo en 2004, est très représentatif de cette posture. Il déclare, dans un entretien donné à l'occasion de l'évènement ${ }^{22}$, vouloir réaliser «des mélanges qui ne sont pas encore calibrés, répertoriés, qui ne sont même pas pensés philosophiquement", afin quill ne soit pas possible, dit-il, de "catégoriser» son travail. Produire de linclassable, au moyen d'une mise en synergie inédite de matériaux dispersés, est ici l'objet même de la création. Cette conception du travail artistique exprime une double intention. À travers le rejet de l'étiquetage et du singularisant, elle manifeste un sentiment, quelque peu océanique, de rattachement au monde, au «toutmonde" (Glissant 1997). Elle marque aussi la volonté de se tenir à l'écart des effets de modes, le refus de la normalisation, de luniformisation et de la récupération marchande.

Elle relève, il est vrai, d’une "esthétique du changement " qui, dans la logique spéculative de l'économie culturelle, peut entrainer la surenchère, la fuite en avant, vers toujours plus dinnovation (Heinich 1998). Dans les milieux les plus compétitifs du marché de l'art, priment la recherche de linouï, du jamais vu, et la fabrique de combinaisons toujours plus inattendues, et souvent productrices de contresens. Car dans ce recyclage forcené et aveugle, la rupture mémorielle est consommée, les pré-contraintes

${ }^{22}$ Cf. L'interview, par Clémentine Aubry, du 25 mai 2004, sur le site http://www.paris-art.com/interv_detail1742.html. 
sont évacuées. Les modèles d'emprunt, détachés de leur contexte et rassemblés dans de nouvelles configurations, perdent leur signification antérieure et changent de connotations. Pour les communautés lointaines, non occidentales, dont ils proviennent, ils peuvent navoir plus aucune pertinence, aucune efficience. La reconversion n'a plus de sens que sur un marché de l'art qui reproduit les relations inégalitaires entre cultures hégémoniques et cultures dominées.

Les études sociologiques le montrent, l'éclectisme contemporain est avant tout le fait d'artistes occidentaux et le reflet des rapports hiérarchiques entre les continents. Alain Quemin, notamment, rappelle que subsiste une forte territorialisation du marché de l'art international, où prévaut encore le "duo-pôle américano-européen", qui ne laisse guère de place à la consécration des artistes des pays périphériques et à leur possibilité d'engagement dans des ouvres d'avant-garde (2002). Ces derniers, hormis ceux qui sont passés par les grandes capitales occidentales ${ }^{23}$, pratiquent somme toute peu l'éclectisme postmoderne... Face à cette réalité, les discours convenus du monde de l'art contemporain sur le métissage et l'ouverture interculturelle révèlent leur facticité. Ils servent en réalité plus souvent de caution à une stratégie d'appropriation que d'aucuns apparentent à du pillage (Feld 2004). De fait, les arts mondialisés, fruits par excellence de la démarche éclectique, sont partie prenante des flux transnationaux et soumis aux termes de l'échange qui régissent la circulation des marchandises entre le Nord et le Sud.

Ils ne sont pas pour autant l'objet de pratiques cyniques, délibérées, d'exploitation. Ils peuvent même être l'expression d'une utopie, lorsque les artistes pensent, à travers eux, faire reconnaître la diversité des cultures et contribuer à leur sauvegarde. Dans les expériences d’art in situ, qui se veulent ancrées dans un site et visent linteraction avec lui ${ }^{24}$, les créateurs mettent en actes cette volonté d'engagement. Cherchant à sỉnsérer au sein de communautés avec lesquelles ils prétendent monter des actions concertées, ils simpliquent dans les politiques patrimoniales et la lutte contre

\footnotetext{
${ }^{23}$ Dans la même étude, Alain Quemin souligne ce fait, page 227, en ces termes : «L'installation dans l'un des pays leaders dans le domaine artistique semble constituer un préalable pour les artistes originaires des pays périphériques au monde de l'art international pour être reconnus par les académies informelles que contrôle, aujourd'hui encore, le monde occidental ».

24 Traduction du Site-Specific Art des auteurs anglo-saxons.
} 
l'hégémonie culturelle nord-américaine et occidentale. Les résultats escomptés de telles entreprises de promotion d'une esthétique relationnelle qui veut re-localiser le geste artistique sont toutefois fréquemment discutables. De fait, les productions réalisées dans le cadre de ces programmes mettent en œuvre des savoirs et des capacités de distanciation, de jeu avec la norme, que ne maitrisent pas toujours les milieux dans lesquels ils sont menés. Dans les sociétés non occidentales où sont conduits certains de ces projets, les artistes et artisans ont une vision traditionaliste de leur art, qui privilégie la répétition immuable des modèles ancestraux, une conception collective de la création, l'anonymat des œuvres. Les innovations qui naissent de ces expériences de partenariat leur paraissent étranges et étrangères. Elles trouvent auprès d'eux peu d'écho, et rejoignent tôt ou tard les circuits habituels de diffusion de lart contemporain, concentrés dans les grandes métropoles des États-Unis, de Grande Bretagne, d'Allemagne ou de France.

Force est ainsi d'admettre que l'éclectisme contemporain répond prioritairement aux attentes des artistes et amateurs d'art des sociétés occidentales, dans leur majorité issus des couches les plus instruites et les plus aisées, sans doute parce qu'il correspond à des dispositions intimement liées à leur mode de vie. Il se pourrait en effet, c'est en tous cas l'hypothèse ici avancée, que le goût du composite et ce quil suppose de concomitances cognitives et sensorielles aient quelque résonance avec les manières modernes d'être et de communiquer.

\section{Une esthétique du fragmentaire}

En recherchant ailleurs et toujours plus loin sa matière, la démarche éclectique qui prévaut aujourd'hui en art dénote un désir de se déprendre de familiarités perceptives, une quête perpétuelle de dépaysement. Elle puise, dans ce but, à plusieurs sources simultanément et, dans cette coalescence de contenus en miettes, développe une esthétique du fragmentaire qui fait écho aux tendances actuelles à la diffraction des comportements, sociaux, intellectuels, esthétiques, émotionnels.

Le mode de production de lart contemporain comme les formes de sa réception ont quelque chose du zapping. Lartiste, et ses publics à sa suite, captent des bribes, déplacent sans cesse le regard, réalisent des synthèses provisoires. Ces opérations cognitives et sensorielles pourraient alors procéder des mêmes mécanismes que cette propension, liée au maniement 
des technologies avancées, à mobiliser plusieurs registres perceptifs et intellectuels en même temps. L'œuvre éclectique convoque une pluralité de références et sadresse à tous les sens à la fois, sur un mode boulimique et synesthésique auquel prédispose la communication de masse moderne. Comme les médias qui diffusent de façon continue des flux d’informations d'origines et de natures différentes, elle multiplie les propositions, dans un même espace-temps, et décuple les capacités d'attention et d'écoute de ses spectateurs. Elle sollicite de la sorte une sensorialité potentiellement totale.

Dans cette mise en scène d'une ubiquité à la fois intellectuelle et sensible, l'éclectisme moderne traduit un changement de comportement. Expression polysémique d'un monde polysémique, il joue de temporalités et de modes de spatialisation faits d'immédiateté et de co-présence, et stimule, en les amplifiant, des expériences cognitives et perceptives transversales, qui sont désormais, dans les sociétés de haute technologie, celles du quotidien.

C'est en cela, aussi, quil défie l'analyse. Car il oblige à revoir les catégories du jugement esthétique, à penser linstable, le composite, et cette vision pluraliste d'un univers en mouvement perpétuel, autant de thèmes de réflexion qui appellent de nouvelles formes d'investigation. La diversification des sources et des angles d'observation, la constitution de corpus inédits et les enquêtes de terrain "multi-sites", sont sans doute parmi les moyens les mieux adaptés pour saisir ces complexités. Elles assurent une traçabilité des œuvres et de leurs auteurs, de leurs sources d’inspiration, qui peut éclairer le processus de création. Mais dans cette démultiplication du regard, dans cet effort pour être partout à la fois, la démarche ethnologique est à l'image de ce qu'elle scrute : elle est éclectique. La démarche, c'est-à-dire l'ensemble des dispositifs d'enquête et d'analyse mis en œuvre dans le travail ethnographique, et non les résultats. Car l'ethnologie ne saurait, pas plus à propos des artefacts et des comportements contemporains que pour tout autre domaine d'exploration, se confondre avec son objet. Dans ce champ comme ailleurs, la distance reste la règle et c'est elle, étayée par cette approche multidirectionnelle, qui doit permettre de dévoiler l'insoupçonnable.

Sans doute est-ce là une orientation inéluctable de la discipline, quel que soit désormais son champ d'étude. Car les mobilités et ces formes d'ubiquité, intellectuelles et perceptives, qui se lisent dans la création contemporaine, valent ailleurs, dans de nombreuses sphères de la vie publique et privée, dont 
elle a aussi à connaître. L'art, et c'est après tout l'une de ses vocations, se fait l'écho de ces réalités, il en est la représentation sublimée, dont l'ethnologie peut se saisir, pour approcher cette logique du fragmentaire et de l'éclectique qui semble s'imposer aujourd'hui. Ce faisant, elle expérimente des protocoles à l'évidence applicables à nombre d'autres de ses objets.

C. C.-B.

\section{Références}

Amselle, Jean-Loup

2005 L'Art de la friche. Essai sur lart contemporain africain. Paris : Flammarion.

Appadurai, Arjun (ed.)

I986 The Social Life of Things. New York : Cambridge.

Bastide, Roger

I970 "Mémoire collective et sociologie du bricolage ", L'Année sociologique zème série, volume 21 : 65-108.

Baudelaire, Charles

I846 Curiosités esthétiques III. Salon de I846, XII, « De l'éclectisme et du doute".

Bensa, Alban \& Eric Fassin

2002 "Les sciences sociales face à l'événement ", Terrain 38, Qu'est-ce qu'un événement?

Capone, Stefania

2007 "Transatlantic Dialogue : Roger Bastide and the African American Religions ", Journal of Religion in Africa 37: I-35.

Certeau, Michel de

1993 La Culture au pluriel. Paris : Points Seuil (I ère édition 1974).

Choron-Baix, Catherine

I983 Molam du Sud Laos réfugiés en France. Thèse de doctorat de $3{ }^{\mathrm{e}}$ cycle en ethnologie, Paris, EHESS, 384 p.

1992 "Jécoute votre voix. Cour d'amour du Laos », in Nicole Revel (ed.), Chants alternés. Asie du Sud-est. Paris : Sudestasie : 8I-99.

Collectif

1979 Rodin et l'Extrême-Orient. Paris : Éditions du Musée Rodin. 
Feld, Steven

2004 «Une si douce berceuse pour la World Music », L'Homme i71-I72 : 389-409.

Freud, Sigmund

1987 Un souvenir d'enfance de Léonard de Vinci. Paris : Gallimard (i ire édition I9IO).

Garnier, Bénédicte (ed.)

2002 Rodin. L'antique est ma jeunesse. Paris : Musée Rodin.

Glissant, Édouard

1997 Traité du Tout-Monde. Paris : Gallimard.

Gruzinski, Serge

2004 Les Quatre Parties du monde. Histoire d'une mondialisation. Paris : La Martinière.

Heinich, Nathalie

${ }_{1998} 8$ Le Triple Jeu de l'art contemporain. Paris : Minuit.

Herkovits, Melville

${ }_{193} 8$ Acculturation. The Study of Cultural Contact. New York : Augustin Publisher.

L'Homme 170

2004 Espèces d'objets.

Leniaud, Jean-Michel

I980 Jean-Baptiste Lassus (I807-I857) ou le temps retrouvé des cathédrales. Paris : Droz.

Lévi-Strauss, Claude I962 La Pensée sauvage. Paris : Plon.

Martinelli, Bruno (ed.)

2005 L'Interrogation du style. Anthropologie, technique et esthétique, Aix-en-Provence : Publications de l'université de Provence.

Mary, André 2000 Le Bricolage africain des héros chrétiens. Paris : Cerf.

Martinet, André (ed.) I968 Le Langage. Paris : Gallimard ("La Pléiade »).

Myers, Fred (ed.) 200I The Empire of Things, Regimes of Value and Material Culture. New Mexico : School of American Research.

Moulin, Raymonde

2003 Le Marché de l'art. Mondialisation et nouvelles technologies. Paris : Champs Flammarion. 
Quemin, Alain

2002 L'Art contemporain international : entre les institutions et le marché (Le rapport disparu). Nîmes : Jacqueline Chambon Artprice.

Shusterman, Richard

I992 L'Art à l'état vif. La pensée pragmatiste et l'esthétique populaire. Paris : Éditions de Minuit.

Tarde, Gabriel

200I Les Lois de limitation. Paris : Les Empêcheurs de tourner en rond ( ${ }^{\text {ère }}$ édition 1890 ).

Touché, Marc

2007 "Muséographier les « musiques électroamplifiées ». Pour une sociohistoire du sonore ", Réseaux 25(I4I-I42) : Sociologies des musiques populaires.

Vilain, Jacques

2002 «Préface » in Bénédicte Garnier (ed.), Rodin. L'antique est ma jeunesse. Paris : Musée Rodin : 4-5. 